Research Article

\title{
Wavelet Analysis of Red Noise and Its Application in Climate Diagnosis
}

\author{
Zhihua Zhang 1 \\ Climate Modeling Laboratory, School of Mathematics, Shandong University, Jinan 250100, China \\ Correspondence should be addressed to Zhihua Zhang; zhangzhihua@sdu.edu.cn
}

Received 21 May 2021; Revised 19 July 2021; Accepted 4 September 2021; Published 27 September 2021

Academic Editor: Neggaz Nabil

Copyright () 2021 Zhihua Zhang. This is an open access article distributed under the Creative Commons Attribution License, which permits unrestricted use, distribution, and reproduction in any medium, provided the original work is properly cited.

Signals are often destroyed by various kinds of noises. A common way to statistically assess the significance of a broad spectral peak in signals and the synchronization between signals is to compare with simple noise processes. At present, wavelet analysis of red noise is studied limitedly and there is no general formula on the distribution of the wavelet power spectrum of red noise. Moreover, the distribution of the wavelet phase of red noise is also unknown. In this paper, for any given real/analytic wavelet, we will use a rigorous statistical framework to obtain the distribution of the wavelet power spectrum and wavelet phase of red noise and apply these formulas in climate diagnosis.

\section{Introduction}

Signals are often destroyed by various kinds of noises during the process of generation, transportation, and processing $[1,2]$. A common way to statistically assess the significance of a broad Fourier/wavelet spectral peak in signals $[3,4]$ and the synchronization between signals is to compare with a white/red noise process $[4,5]$. White noise has zero mean, has constant variance, and is uncorrelated in time. As its name suggests, white noise has a power spectrum which is uniformly spread across all allowable frequencies. Different from white noise, red noise has a power spectrum weighted toward low frequencies and is serially correlated in time. Red noise can describe climatic background noise with relatively enhanced low-frequency fluctuations arising from the interaction of white noise forcing with the slow-response components in the earth system (e.g., the thermal inertia of the oceans provides memory, effectively integrating atmospheric "weather" forcing [6]). In practice, the red noise model has always provided a reasonable description of the noise spectra for a variety of climatic and hydrological time series [5-9]. The red noise is also an important noise model in outputs of the feedback system [4], the neural network coupled with genetic algorithm [10], the optimization system in random scenarios $[11,12]$, and the big data processing system $[5,13]$.
Wavelet analysis is a very popular analysis tool for a wide range of applications, including time-frequency analysis, feature extraction, statistical estimation, and denoising. A wavelet is a waveform-like function which has zero mean and is localized in both time and frequency space. The wavelet transform is the set of inner products of all dilated and translated wavelets with a signal; in detail, the wavelet transform of a discrete signal $X=\left\{x_{k}\right\}_{k=0, \ldots, N-1}$ with time step $\delta t$ is defined as follows $[14,15]$ :

$$
W_{n}(s)=\sum_{m=0}^{N-1} x_{m} \bar{\psi}\left(\frac{(m-n) \delta t}{s}\right)
$$

where $\bar{\psi}$ is the conjugate of $\psi \cdot\left|W_{n}(s)\right|^{2}$ is called the wavelet power spectrum, and $\operatorname{Arg}\left(W_{n}(s)\right)$ is called the wavelet phase. Due to time and frequency localization of the wavelet, the wavelet transform can extract localized intermittent periodicity of any signal very well.

Wavelet analysis of white noise has been widely studied (e.g., [16-19]), while wavelet analysis of red noise is studied limitedly, only using some specific wavelets (Morlet/Paul/DOG wavelets [15] and modulated Haar wavelet [20]). Since 1980s, a large family of wavelets with nice properties has been constructed [14]. However, there is no general formula on the distribution of the wavelet power spectrum of red noise. On the other hand, the wavelet phase can be used to test the 
synchronization between signals, but the distribution of the wavelet phase of red noise is also unknown. In this paper, for any given real/analytic wavelet, we will use a rigorous statistical framework to derive a general formula for the distribution of the wavelet power spectrum and wavelet phase of red noise.

\section{Background}

The simplest red noise model is the lag-1 autoregressive (AR (1)) red noise process $[1,4-6,15]$ : Let $X=\left\{x_{k}\right\}_{k=0,1, \ldots, N-1}$ with time step $\delta t$ be an AR (1) red noise with parameters $\lambda(0 \leq \lambda<1)$ and $\sigma^{2}$; then,

$$
\begin{aligned}
& x_{0}=0, \\
& x_{k}=\lambda x_{k-1}+Z_{k}, \quad k \in \mathbb{Z}_{+},
\end{aligned}
$$

where $\left\{Z_{k}\right\}_{k \in \mathbb{Z}_{+}}$is a Gaussian white noise with mean 0 and variance $\sigma^{2}$ and $E\left[Z_{k} Z_{l}\right]=0(k \neq l)$. The discrete Fourier transform of an AR (1) red noise is

$$
\widehat{x}_{k}=\frac{1}{N} \sum_{n=0}^{N-1} x_{n} e^{-i n \theta_{k}}, \quad k=0,1, \ldots, N-1,
$$

where $\theta_{k}=(2 \pi k / N)$. Again, by equation (1), the wavelet transform of an AR (1) red noise can be expressed as [15]

$$
W_{n}(s)=\sum_{k=0}^{N-1} \widehat{x}_{k} \overline{\widehat{\psi}}\left(s \omega_{k}\right) e^{i n \theta_{k}}
$$

where $\omega_{k}=\left(\theta_{k} / \delta t\right), k \leq(N / 2)$, and $\omega_{k}=-\left(\theta_{N-k} / \delta t\right), k>$ $(N / 2)$ (here, we have fixed some typos according to Torrence and Compo's Matlab codes [15]).
Torrence and Compo [15] gave an empirical distribution of the Morlet wavelet power spectrum of an AR (1) red noise:

$$
\left|W_{n}(s)\right|^{2} \text { is distributed as } \frac{\left(1-\lambda^{2}\right) \widetilde{\sigma}^{2}}{2\left(1-2 \lambda \cos (2 \pi k / N)+\lambda^{2}\right)} \chi_{2}^{2} \text {, }
$$

where $\chi_{2}^{2}$ is the chi-square distribution with two degrees of freedom and $\tilde{\sigma}^{2}$ is the variance of the AR (1) red noise $\left(\widetilde{\sigma}^{2} \approx \sigma^{2} /\left(1-\lambda^{2}\right)\right)$. For Paul/DOG wavelet power spectrum of an AR (1) red noise, Torrence and Compo [15] empirically gave the following:

$$
\left|W_{n}(s)\right|^{2} \text { is distributed as } \frac{\left(1-\lambda^{2}\right) \tilde{\sigma}^{2}}{1-2 \lambda \cos (2 \pi k / N)+\lambda^{2}} \chi_{1}^{2} \text {. }
$$

Formulas (5) and (6) are empirically obtained by assuming that "the wavelet spectrum of a red noise is distributed as its Fourier spectrum" [15].

Zhang and Jorgensen [20] showed that under the rigorous statistical framework, the modulated Haar wavelet power spectra of AR (1) red noise is distributed as

$$
\frac{2 \sigma^{2}}{s\left(1-\alpha^{2}\right)}\left(\sigma_{R}^{2} \chi_{1}^{2}+\sigma_{I}^{2} \chi_{2}^{2}\right)
$$

where $\chi_{1}$ and $\chi_{2}$ are two independent standard Gaussian distributions. $\quad$ Also, $\quad P_{k}(\lambda)=\left(1-\lambda^{2}\right) /(1-2 \lambda \cos$ $\left.(4 k \pi / s)+\lambda^{2}\right)$ and

$$
\begin{aligned}
& \sigma_{R}^{2}=\frac{s}{2} P_{k}(\lambda)+\frac{4 \lambda\left(1-\lambda^{(s / 2)}\right)}{\left(1-\lambda^{2}\right)}\left(\lambda-\cos \frac{4 k \pi}{s}\right)\left(1-\lambda \cos \frac{2 k \pi}{s^{*}}\right) P_{k}^{2}(\lambda)-\frac{2 \lambda^{2}\left(1-\lambda^{s / 2}\right)^{2}}{\left(1-\lambda^{2}\right)}\left(\lambda-\cos \frac{4 k \pi}{s}\right)^{2} P_{k}^{2}(\lambda), \\
& \sigma_{I}^{2}=\frac{s}{2} P_{k}(\lambda)+P_{k}^{2}(\lambda) \frac{4 \lambda^{2}\left(1-\lambda^{s / 2}\right)}{\left(1-\lambda^{2}\right)^{2}} \sin ^{2} \frac{4 k \pi}{s}-P_{k}^{2}(\lambda) \frac{2 \lambda^{2}\left(1-\lambda^{s / 2}\right)^{2}}{\left(1-\lambda^{2}\right)^{2}} \sin ^{2} \frac{4 k \pi}{s}
\end{aligned}
$$

Until now, wavelet analysis of red noise is studied limitedly, only using some specific wavelets. There is no general formula on the distribution of the wavelet power spectrum and wavelet phase of an AR (1) red noise.

\section{Main Results}

We will use a rigorous statistical framework to establish the distribution of the wavelet power spectrum and wavelet phase of an AR (1) red noise with time step $\delta t$, length $N$, and parameters $\lambda$ and $\sigma^{2}$. Here, we assume a very weak condition: $\lambda^{N} \approx 0$ and $\left(1 / N^{2}\right) \approx 0$. In practice, all climatic background noises satisfy this condition, e.g., if $\lambda=0.5$ and $N=50$, then $\lambda^{N} \approx 10^{-8}$ and $\left(1 / N^{2}\right)=0.0004$.

A wavelet is real if it is a real-valued function. All of spline wavelets and most of compactly supported wavelets are real wavelets.
Theorem 1. For any real wavelet, the wavelet power spectrum $\left|W_{n}(s)\right|^{2}$ of an AR (1) red noise is distributed as

$$
\left(\frac{2 \sigma^{2}}{N} \sum_{k=0}^{[N / 2]} \frac{\left|\widehat{\psi}\left(s \omega_{k}\right)\right|^{2}}{1-2 \lambda \cos \theta_{k}+\lambda^{2}}\right) \chi_{1}^{2} \text {. }
$$

A wavelet $\psi$ is said to be analytic if its Fourier transform satisfies $\widehat{\psi}(\omega)=0, \omega<0$. Different from real wavelets, analytic wavelets can extract not only spectral information but also phase information from any given signal.

Theorem 2. For any analytic wavelet, the wavelet power spectrum of an AR (1) red noise is distributed as

$$
\left(\frac{\sigma^{2}}{2 N} \sum_{k=0}^{[N / 2]} \frac{\left|\widehat{\psi}\left(s \omega_{k}\right)\right|^{2}}{1-2 \lambda \cos \theta_{k}+\lambda^{2}}\right) \chi_{2}^{2} \text {. }
$$


Theorem 3. For any analytic wavelet, the wavelet phase of an $A R$ (1) red noise is distributed uniformly on $[0,2 \pi]$.

\section{Proofs}

The $\operatorname{AR}(1)$ red noise with parameters $\lambda,|\lambda|<1$ and $\sigma^{2}$ can be expressed as

$$
x_{k}=\sum_{\nu=1}^{k} \lambda^{k-v} Z_{v}, \quad k \in \mathbb{Z}_{+} .
$$

By $E\left[Z_{\nu}\right]=0$,

$$
E\left[x_{k}\right]=\sum_{\nu=1}^{k} \lambda^{k-v} E\left[Z_{\nu}\right]=0,
$$

and so the real part of the wavelet transform is

$$
\operatorname{Re}\left(W_{n}(s)\right)=\sum_{m=0}^{N-1} x_{m} \operatorname{Re}\left(\psi\left(\frac{(m-n) \delta t}{s}\right)\right)
$$

Since $\operatorname{Re}\left(W_{n}(s)\right)$ is a linear combination of $\left\{x_{m}\right\}$, $\operatorname{Re}\left(W_{n}(s)\right)$ is a Gaussian random variable and

$$
\begin{aligned}
& E\left[\operatorname{Re}\left(W_{n}(s)\right)\right]=0, \\
& \operatorname{Var}\left(\operatorname{Re}\left(W_{n}(s)\right)\right)=E\left[\left(\operatorname{Re}\left(W_{n}(s)\right)\right)^{2}\right] .
\end{aligned}
$$

Similarly, $\operatorname{Im}\left(W_{n}(s)\right)$ is a Gaussian random variable and

$$
\begin{aligned}
& E\left[\operatorname{Im}\left(W_{n}(s)\right)\right]=0, \\
& \operatorname{Var}\left(\operatorname{Im}\left(W_{n}(s)\right)\right)=E\left[\left(\operatorname{Im}\left(W_{n}(s)\right)\right)^{2}\right] .
\end{aligned}
$$

Denote $\operatorname{Arg}\left(\overline{\widehat{\psi}}\left(s \omega_{k}\right)\right)=\varphi_{k} . \quad$ Then, $\quad \overline{\widehat{\psi}}\left(s \omega_{k}\right) e^{i n \theta_{k}}=\mid \widehat{\psi}$ $\left(s \omega_{k}\right) \mid e^{i \eta_{n, k}}$, where $\eta_{n, k}=\varphi_{k}+n \theta_{k}$. By equation (4), we have

$$
\begin{aligned}
\operatorname{Re}\left(W_{n}(s)\right) & =\sum_{k=0}^{N-1}\left|\widehat{\psi}\left(s \omega_{k}\right)\right| \operatorname{Re}\left(\widehat{x}_{k} e^{i \eta_{n, k}}\right)=\sum_{k=0}^{N-1}\left|\widehat{\psi}\left(s \omega_{k}\right)\right|\left(\operatorname{Re}\left(\widehat{x}_{k}\right) \cos \eta_{n, k}-\operatorname{Im}\left(\widehat{x}_{k}\right) \sin \eta_{n, k}\right), \\
\operatorname{Im}\left(W_{n}(s)\right) & =\sum_{k=0}^{N-1}\left|\widehat{\psi}\left(s \omega_{k}\right)\right|\left(\operatorname{Re}\left(\widehat{x}_{k}\right) \sin \eta_{n, k}+\operatorname{Im}\left(\widehat{x}_{k}\right) \cos \eta_{n, k}\right) .
\end{aligned}
$$

Let

$$
\begin{aligned}
& \alpha_{k, j}=E\left[\operatorname{Re}\left(\hat{x}_{k}\right) \operatorname{Re}\left(\hat{x}_{j}\right)\right], \\
& \beta_{k, j}=E\left[\operatorname{Re}\left(\hat{x}_{k}\right) \operatorname{Im}\left(\hat{x}_{j}\right)\right], \\
& \gamma_{k, j}=E\left[\operatorname{Im}\left(\hat{x}_{k}\right) \operatorname{Im}\left(\hat{x}_{j}\right)\right] .
\end{aligned}
$$

$$
\begin{aligned}
& \operatorname{Var}\left(\operatorname{Re}\left(W_{n}(s)\right)\right) \\
& =\sum_{k, j=0}^{N-1}\left|\widehat{\psi}\left(s \omega_{j}\right) \widehat{\psi}\left(s \omega_{j}\right)\right|\left(\alpha_{k, j} \cos \eta_{n, k} \cos \eta_{n, j}-\beta_{k, j} \sin \eta_{n, k} \cos \eta_{n, j}-\beta_{j, k} \cos \eta_{n, k} \sin \eta_{n, j}+\gamma_{k, j} \sin \eta_{n, k} \sin \eta_{n, j}\right), \\
& \operatorname{Var}\left(\operatorname{Im}\left(W_{n}(s)\right)\right) \\
& =\sum_{k, j=0}^{N-1}\left|\widehat{\psi}\left(s \omega_{j}\right) \widehat{\psi}\left(s \omega_{j}\right)\right|\left(\alpha_{k, j} \sin \eta_{n, k} \sin \eta_{n, j}+\beta_{k, j} \cos \eta_{n, k} \sin \eta_{n, j}+\beta_{j, k} \sin \eta_{n, k} \sin \eta_{n, j}+\gamma_{k, j} \cos \eta_{n, k} \cos \eta_{n, j}\right), \\
& E\left[\operatorname{Re}\left(W_{n}(s)\right) \operatorname{Im}\left(W_{n}(s)\right)\right] \\
& =\sum_{k, j=0}^{N-1}\left|\widehat{\psi}\left(s \omega_{k}\right) \widehat{\psi}\left(s \omega_{j}\right)\right|\left(\alpha_{k, j} \cos \eta_{n, k} \sin \eta_{n, j}+\beta_{k, j} \sin \eta_{n, k} \sin \eta_{n, j}-\beta_{j, k} \cos \eta_{n, k} \cos \eta_{n, j}-\gamma_{k, j} \sin \eta_{n, k} \cos \eta_{n, j}\right) .
\end{aligned}
$$

From these, we see that the computations of variances and the correlation of $\operatorname{Re}\left(W_{n}(s)\right)$ and $\operatorname{Im}\left(W_{n}(s)\right)$ are reduced to the computations of $\alpha_{k, j}, \beta_{k, j}$, and $\gamma_{k, j}$.
4.1. Computation of $\alpha_{k, j}$. By equation (3), we have

$$
\operatorname{Re}\left(\hat{x}_{k}\right)=\frac{1}{N} \sum_{n=0}^{N-1} x_{n} \cos \left(n \theta_{k}\right)
$$


where $\theta_{k}=(2 \pi k / N)$. Furthermore,

$$
E\left[\operatorname{Re}\left(\widehat{x}_{j}\right) \operatorname{Re}\left(\widehat{x}_{k}\right)\right]=\frac{1}{N^{2}} \sum_{l, p=1}^{N-1} E\left[x_{l} x_{p}\right] \cos \left(l \theta_{j}\right) \cos \left(p \theta_{k}\right) .
$$

By equation (11), it follows that

$$
x_{l} x_{p}=\left(\sum_{\nu=1}^{l} \lambda^{l-v} Z_{\nu}\right)\left(\sum_{m=1}^{p} \lambda^{p-m} Z_{m}\right)=\sum_{\nu=1}^{l} \sum_{m=1}^{p} \lambda^{l+p-\nu-m} Z_{\nu} Z_{m}
$$

where

$$
E\left[Z_{v} Z_{m}\right]= \begin{cases}0, & n u \neq m \\ \sigma^{2}, & n \mathcal{u}=m .\end{cases}
$$

Hence, we have for $l>p$,

$$
E\left[x_{l} x_{p}\right]=\sum_{\nu=1}^{p} \sum_{m=1}^{p} \lambda^{l+p-\nu-m} E\left[Z_{\nu} Z_{m}\right]=\sigma^{2} \sum_{\nu=1}^{p} \lambda^{l+p-2 v}=\sigma^{2} \frac{\lambda^{l+p}-\lambda^{l-p}}{\lambda^{2}-1} .
$$

Similarly, for $l \leq p$,

$$
E\left[x_{l} x_{p}\right]=\sigma^{2} \frac{\lambda^{l+p}-\lambda^{p-l}}{\lambda^{2}-1} .
$$

From these, we obtain

$$
N^{2}\left(\frac{\lambda^{2}-1}{\sigma^{2}}\right) E\left[\operatorname{Re}\left(\widehat{x}_{k}\right) \operatorname{Re}\left(\widehat{x}_{j}\right)\right]=\Sigma_{1}+\Sigma_{2},
$$

where

$$
\begin{aligned}
& \Sigma_{1}=\sum_{l=1}^{N-1} \lambda^{l} \cos \left(l \theta_{j}\right) \sum_{p=1}^{l-1}\left(\lambda^{p}-\lambda^{-p}\right) \cos \left(p \theta_{k}\right), \\
& \Sigma_{2}=\sum_{l=1}^{N-1}\left(\lambda^{l}-\lambda^{-l}\right) \cos \left(l \theta_{j}\right) \sum_{p=l}^{N-1} \lambda^{p} \cos \left(p \theta_{k}\right) .
\end{aligned}
$$

We first compute $\Sigma_{1}$. By using Euler formula $\cos \left(p \theta_{k}\right)=\operatorname{Re}\left(e^{i p \theta_{k}}\right)$, the inner summation becomes

$$
\sum_{p=1}^{l-1}\left(\lambda^{p}-\lambda^{-p}\right) \cos \left(p \theta_{k}\right)=P_{k}(\lambda)+\mu_{l}^{1}+\mu_{l}^{2}
$$

where $P_{k}(\lambda)=\left(1-\lambda^{2}\right) /\left(1-2 \lambda \cos \theta_{k}+\lambda^{2}\right)$ and

$$
\begin{aligned}
& \mu_{l}^{1}=-\frac{\lambda^{l}}{1-\lambda^{2}} P_{k}(\lambda)\left(\cos \left(l \theta_{k}\right)-\lambda \cos (l-1) \theta_{k}\right), \\
& \mu_{l}^{2}=\frac{\lambda^{-l+1}}{1-\lambda^{2}} P_{k}(\lambda)\left(\lambda \cos \left(l \theta_{k}\right)-\cos (l-1) \theta_{k}\right) .
\end{aligned}
$$

From this and equation (25), we obtain

$$
\Sigma_{1}=P_{k}(\lambda) \sum_{l=1}^{N-1} \lambda^{l} \cos \left(l \theta_{j}\right)+\sum_{l=1}^{N-1} \lambda^{l} \cos \left(l \theta_{j}\right) \mu_{l}^{1}+\sum_{l=1}^{N-1} \lambda^{l} \cos \left(l \theta_{j}\right) \mu_{l}^{2}=: \Sigma_{11}+\Sigma_{12}+\Sigma_{13} .
$$

For $\Sigma_{11}$, when $\lambda^{N} \approx 0$,

For $\Sigma_{13}$, we have

$$
\Sigma_{11}=P_{k}(\lambda) \frac{\lambda \cos \theta_{j}-\lambda^{2}}{1-2 \lambda \cos \theta_{j}+\lambda^{2}}
$$

$$
\Sigma_{13}=\frac{\lambda^{2}}{1-2 \lambda \cos \theta_{k}+\lambda^{2}} \sum_{l=1}^{N-1}\left(\cos \left(l \theta_{k}\right)-\lambda^{-1} \cos (l-1) \theta_{k}\right) \cos \left(l \theta_{j}\right)=\frac{A(\lambda)}{2\left(1-2 \lambda \cos \theta_{k}+\lambda^{2}\right)},
$$

where 


$$
A(\lambda)=\lambda^{2} \sum_{l=1}^{N-1}\left[\cos \left(l \theta_{k+j}\right)+\cos \left(l \theta_{k-j}\right)-\lambda^{-1}\left(\cos \left(l \theta_{k+j}-\theta_{k}\right)+\cos \left(l \theta_{k-j}-\theta_{k}\right)\right)\right] .
$$

Noticing that $\theta_{k}=(2 \pi k / N)$, we consider four cases to compute $A(\lambda)$ and then estimate $\Sigma_{13}$ :

(i) For $k \neq j$ and $k+j \neq N$, by $\theta_{k}=(2 \pi k / N)$ and $\sum_{l=0}^{N-1} \cos \left(l \theta_{k}\right)=\operatorname{Re}\left(\sum_{l=0}^{N-1} e^{i l \theta_{k}}\right)=0$, we have

$$
A(\lambda)=-2 \lambda^{2}+2 \lambda \cos \theta_{k}
$$
and then $\Sigma_{13}=\left(-\lambda^{2}+\lambda \cos \theta_{k}\right) /(1-2 \lambda \cos$ $\left.\theta_{k}+\lambda^{2}\right)$.

(ii) For $k=j \neq(N / 2)$, we obtain

$$
A(\lambda)=\lambda^{2} \sum_{l=1}^{N-1}\left(\cos \left(2 l \theta_{k}\right)+1-\lambda^{-1}\left(\cos \left(2 l \theta_{k}-\theta_{k}\right)+\cos \theta_{k}\right)\right)
$$

and then

$$
\Sigma_{13}=\frac{(N-2)\left(\lambda^{2}-\lambda \cos \theta_{k}\right)}{2\left(1-2 \lambda \cos \theta_{k}+\lambda^{2}\right)}
$$

$$
A(\lambda)=(N-2)\left(\lambda^{2}-\lambda \cos \theta_{k}\right) .
$$

Therefore,

(iii) For $k+j=N$ and $k \neq j$, we obtain

$$
A(\lambda)=\lambda^{2} \sum_{l=1}^{N-1}\left(1+\cos \left(l \theta_{k-j}\right)-\lambda^{-1}\left(\cos \theta_{k}+\cos \left(l \theta_{k-j}-\theta_{k}\right)\right)\right)=(N-2)\left(\lambda^{2}-\lambda \cos \theta_{k}\right)
$$

Therefore,

$$
\Sigma_{13}=\frac{(N-2)\left(\lambda^{2}-\lambda \cos \theta_{k}\right)}{2\left(1-2 \lambda \cos \theta_{k}+\lambda^{2}\right)} .
$$

(iv) For $k=j=(N / 2)$, we obtain

$$
A(\lambda)=\lambda^{2} \sum_{l=1}^{N-1}\left(2-\frac{2 \cos \theta_{k}}{\lambda}\right)=2(N-1)\left(\lambda^{2}-\lambda \cos \theta_{k}\right) \text {, }
$$

$$
\Sigma_{13}=\frac{(N-1)\left(\lambda^{2}-\lambda \cos \theta_{k}\right)}{1-2 \lambda \cos \theta_{k}+\lambda^{2}}
$$

Now, we compute $\Sigma_{2}$ in equation (25). When $\lambda^{N} \approx 0$, it follows that

$$
\sum_{p=l}^{N-1} \lambda^{p} \cos \left(p \theta_{k}\right)=\frac{\lambda^{l} \cos \left(l \theta_{k}\right)-\lambda^{l+1} \cos (l-1) \theta_{k}}{1-2 \lambda \cos \theta_{k}+\lambda^{2}} .
$$

and so

$$
\Sigma_{2}=\sum_{l=1}^{N-1}\left(\lambda^{l}-\lambda^{-l}\right) \cos \left(l \theta_{j}\right)\left(\lambda \frac{\cos \left(l \theta_{k}\right)-\lambda \cos (l-1) \theta_{k}}{1-2 \lambda \cos \theta_{k}+\lambda^{2}}\right)=\Sigma_{21}+\Sigma_{22}
$$

where

$$
\begin{aligned}
& \Sigma_{21}=\frac{1}{1-2 \lambda \cos \theta_{k}+\lambda^{2}} \sum_{l=1}^{N-1} \lambda^{2 l} \cos \left(l \theta_{j}\right)\left(\cos \left(l \theta_{k}\right)-\lambda \cos (l-1) \theta_{k}\right), \\
& \Sigma_{22}=-\frac{1}{1-2 \lambda \cos \theta_{k}+\lambda^{2}} \sum_{l=1}^{N-1} \cos \left(l \theta_{j}\right)\left(\cos \left(l \theta_{k}\right)-\lambda \cos (l-1) \theta_{k}\right) .
\end{aligned}
$$


Furthermore,

$$
\Sigma_{22}=-\frac{1}{2\left(1-2 \lambda \cos \theta_{k}+\lambda^{2}\right)} \sum_{l=1}^{N-1}\left(\left(\cos \left(l \theta_{k+j}\right)+\cos \left(l \theta_{k-j}\right)\right)-\lambda\left(\cos \left(l \theta_{k+j}-\theta_{k}\right)+\cos \left(l \theta_{k-j}-\theta_{k}\right)\right)\right) .
$$

Similar to the estimation process of $\Sigma_{13}$, we have

$$
\begin{aligned}
& \Sigma_{22}=\frac{2-2 \lambda \cos \theta_{k}}{2\left(1-2 \lambda \cos \theta_{k}+\lambda^{2}\right)}, \quad k \neq j \&, k+j \neq N, \\
& \Sigma_{22}=-\frac{(N-2)\left(1-\lambda \cos \theta_{k}\right)}{2\left(1-2 \lambda \cos \theta_{k}+\lambda^{2}\right)}, \quad k=j \neq \frac{N}{2}, \\
& \Sigma_{22}=-\frac{(N-2)\left(1-\lambda \cos \theta_{k}\right)}{2\left(1-2 \lambda \cos \theta_{k}+\lambda^{2}\right)}, \quad k+j=N \&, k \neq j, \\
& \Sigma_{22}=-\frac{(N-1)\left(1-\lambda \cos \theta_{k}\right)}{1-2 \lambda \cos \theta_{k}+\lambda^{2}}, \quad k=j=\frac{N}{2} .
\end{aligned}
$$

Combining this and equations (29)-(42), noticing that $\Sigma_{12}+\Sigma_{21}=0$, it follows that

$$
\begin{aligned}
& \Sigma_{1}+\Sigma_{2}=P_{k}(\lambda) P_{j}(\lambda), \quad k \neq j \&, k+j \neq N, \\
& \Sigma_{1}+\Sigma_{2}=-\frac{N\left(1-\lambda^{2}\right)}{2\left(1-2 \lambda \cos \theta_{k}+\lambda^{2}\right)}+P_{k}(\lambda)\left(\frac{\lambda \cos \theta_{j}-\lambda^{2}}{1-2 \lambda \cos \theta_{j}+\lambda^{2}}-1\right), \quad k=j \neq \frac{N}{2}, \\
& \Sigma_{1}+\Sigma_{2}=-\frac{N\left(1-\lambda^{2}\right)}{2\left(1-2 \lambda \cos \theta_{k}+\lambda^{2}\right)}+P_{k}(\lambda)\left(\frac{\lambda \cos \theta_{j}-\lambda^{2}}{1-2 \lambda \cos \theta_{j}+\lambda^{2}}-1\right), \quad k+j=N \&, k \neq j, \\
& \Sigma_{1}+\Sigma_{2}=-\frac{N\left(1-\lambda^{2}\right)}{1-2 \lambda \cos \theta_{k}+\lambda^{2}}+P_{k}(\lambda)\left(\frac{\lambda \cos \theta_{j}-\lambda^{2}}{1-2 \lambda \cos \theta_{j}+\lambda^{2}}-1\right), \quad k=j=\frac{N}{2} .
\end{aligned}
$$
have

Noticing $|\lambda|<1$ and $1-2 \lambda \cos \theta_{k}+\lambda^{2}>(1-|\lambda|)^{2}$, we 


$$
\begin{array}{r}
\left|P_{k}(\lambda) P_{j}(\lambda)\right|<\frac{4}{(1-|\lambda|)^{4}}, \\
\left|P_{k}(\lambda)\left(\frac{\lambda \cos \theta_{j}-\lambda^{2}}{1-2 \lambda \cos \theta_{j}+\lambda^{2}}-1\right)\right|<\frac{12}{(1-|\lambda|)^{4}} .
\end{array}
$$

Again, by equation (25), we obtain

$$
\begin{gathered}
\left|E\left[\operatorname{Re}\left(\hat{x}_{k}\right) \operatorname{Re}\left(\hat{x}_{j}\right)\right]\right| \leq \frac{C}{N^{2}}, \quad k \neq j \& k+j \neq N, \\
\left|E\left[\operatorname{Re}\left(\hat{x}_{k}\right) \operatorname{Re}\left(\hat{x}_{j}\right)\right]-\frac{\sigma^{2}}{2 N\left(1-2 \lambda \cos \theta_{k}+\lambda^{2}\right)}\right| \leq \frac{C}{N^{2}}, \quad k=j \neq \frac{N}{2} \text { or } k+j=N \& k \neq j, \\
\left|E\left[\operatorname{Re}\left(\hat{x}_{k}\right) \operatorname{Re}\left(\hat{x}_{j}\right)\right]-\frac{\sigma^{2}}{N\left(1-2 \lambda \cos \theta_{k}+\lambda^{2}\right)}\right| \leq \frac{C}{N^{2}}, \quad k=j=\frac{N}{2},
\end{gathered}
$$

where $C=12 \sigma^{2} /\left(1-|\lambda|^{2}\right)(1-|\lambda|)^{4}$ is a constant.

Finally, we obtain the following.
Lemma 1. When $\lambda^{N} \approx 0$ and $\left(1 / N^{2}\right) \approx 0$, the series $\left\{\operatorname{Re}\left(\widehat{x}_{k}\right)\right\}_{k=0, \ldots, N-1}$ satisfy

$$
\alpha_{k, j}=E\left[\operatorname{Re}\left(\hat{x}_{k}\right) \operatorname{Re}\left(\hat{x}_{j}\right)\right]= \begin{cases}0, & k \neq j \text { and } k+j \neq N, \\ \frac{\sigma^{2}}{2 N\left(1-2 \lambda \cos \theta_{k}+\lambda^{2}\right)}, & k=j \neq \frac{N}{2} \text { or } k+j=N \& k \neq j, \\ \frac{\sigma^{2}}{N\left(1-2 \lambda \cos \theta_{k}+\lambda^{2}\right)}, & k=j=\frac{N}{2} .\end{cases}
$$

4.2. Computation of $\gamma_{k, j}$. By equation (3), we have

Similar to the deducing process of equation (25), we have $E\left[\operatorname{Im}\left(\widehat{x}_{j}\right) \operatorname{Im}\left(\widehat{x}_{k}\right)\right]=\frac{1}{N^{2}} \sum_{l, p=1}^{N-1} E\left[x_{l} x_{p}\right] \sin \left(l \theta_{j}\right) \sin \left(p \theta_{k}\right)$.

$$
\begin{aligned}
& N^{2}\left(\frac{\lambda^{2}-1}{\sigma^{2}}\right) E\left[\operatorname{Im}\left(\widehat{x}_{j}\right) \operatorname{Im}\left(\hat{x}_{k}\right)\right] \\
& =\sum_{l=1}^{N-1} \lambda^{l} \sin \left(l \theta_{j}\right) \sum_{p=1}^{l-1}\left(\lambda^{p}-\lambda^{-p}\right) \sin \left(p \theta_{k}\right)+\sum_{l=1}^{N-1}\left(\lambda^{l}-\lambda^{-l}\right) \sin \left(l \theta_{j}\right) \sum_{p=l}^{N-1} \lambda^{p} \sin \left(p \theta_{k}\right) \\
& =: \widetilde{\Sigma}_{1}+\widetilde{\Sigma}_{2} .
\end{aligned}
$$

Noticing that 


$$
\sum_{p=1}^{l-1} \lambda^{p} \sin \left(p \theta_{k}\right)=\frac{-\lambda^{l} \sin \left(l \theta_{k}\right)+\lambda \sin \theta_{k}+\lambda^{l+1} \sin (l-1) \theta_{k}}{1-2 \lambda \cos \theta_{k}+\lambda^{2}},
$$

we obtain

$$
\sum_{p=1}^{l-1}\left(\lambda^{p}-\lambda^{-p}\right) \sin \left(p \theta_{k}\right)=\widetilde{\mu}_{l}^{1}+\widetilde{\mu}_{l}^{2}
$$

where

$$
\begin{aligned}
& \tilde{\mu}_{l}^{1}=-\lambda^{\lambda^{l}} \frac{\sin \left(l \theta_{k}\right)-\lambda \sin (l-1) \theta_{k}}{1-2 \lambda \cos \theta_{k}+\lambda^{2}}, \\
& \widetilde{\mu}_{l}^{2}=\lambda^{-l+2} \frac{\sin \left(l \theta_{k}\right)-\lambda^{-1} \sin (l-1) \theta_{k}}{1-2 \lambda \cos \theta_{k}+\lambda^{2}} .
\end{aligned}
$$

\section{Notice that}

$$
\widetilde{\Sigma}_{1}=\sum_{l=1}^{N-1} \lambda^{l} \tilde{\mu}_{l}^{1} \sin \left(l \theta_{j}\right)+\sum_{l=1}^{N-1} \lambda^{l} \widetilde{\mu}_{l}^{2} \sin \left(l \theta_{j}\right)=: \widetilde{\Sigma}_{11}+\widetilde{\Sigma}_{12}
$$

$$
\widetilde{\Sigma}_{12}=\frac{\lambda^{2}}{2\left(1-2 \lambda \cos \theta_{k}+\lambda^{2}\right)} \sum_{l=1}^{N-1}\left(\left(-\cos \left(l \theta_{k+j}\right)+\cos \left(l \theta_{k-j}\right)\right)+\frac{1}{\lambda}\left(\cos \left(l \theta_{k+j}-\theta_{k}\right)-\cos \left(l \theta_{k-j}-\theta_{k}\right)\right)\right) .
$$

For $k \neq j$ and $k+j \neq N$, we have

$$
\sum_{l=0}^{N-1} \cos \left(l \theta_{k+j}\right)=\sum_{l=0}^{N-1} \cos \left(l \theta_{k-j}\right)=\sum_{l=0}^{N-1} \cos \left(l \theta_{k+j}-\theta_{k}\right)=\sum_{l=0}^{N-1} \cos \left(l \theta_{k-j}-\theta_{k}\right)=0
$$

and so $\widetilde{\Sigma}_{12}=0$.

For $k=j \neq(N / 2)$, we have

$$
\tilde{\Sigma}_{12}=\frac{N\left(\lambda^{2}-\lambda \cos \theta_{k}\right)}{2\left(1-2 \lambda \cos \theta_{k}+\lambda^{2}\right)} .
$$

For $k+j=N$ and $k \neq(N / 2)$, we have

$$
\tilde{\Sigma}_{12}=-\frac{N\left(\lambda^{2}-\lambda \cos \theta_{k}\right)}{2\left(1-2 \lambda \cos \theta_{k}+\lambda^{2}\right)} \text {. }
$$

For $k=j=(N / 2), \widetilde{\Sigma}_{12}=0$. that

We estimate $\widetilde{\Sigma}_{2}$ in equation (51). Since $\lambda^{N} \approx 0$, it follows

$$
\sum_{p=l}^{N-1} \lambda^{p} \sin \left(p \theta_{k}\right)=\lambda^{l} \frac{\sin \left(l \theta_{k}\right)-\lambda \sin (l-1) \theta_{k}}{1-2 \lambda \cos \theta_{k}+\lambda^{2}}
$$

Furthermore,

$$
\begin{aligned}
\widetilde{\Sigma}_{2}= & \frac{1}{1-2 \lambda \cos \theta_{k}+\lambda^{2}} \sum_{l=1}^{N-1} \lambda^{2 l} \sin \left(l \theta_{j}\right)\left(\sin \left(l \theta_{k}\right)-\lambda \sin (l-1) \theta_{k}\right) \\
& -\frac{1}{1-2 \lambda \cos \theta_{k}+\lambda^{2}} \sum_{l=1}^{N-1} \sin \left(l \theta_{j}\right)\left(\sin \left(l \theta_{k}\right)-\lambda \sin (l-1) \theta_{k}\right) \\
= & \widetilde{\Sigma}_{21}+\widetilde{\Sigma}_{22} .
\end{aligned}
$$

By equation (55), it follows that $\widetilde{\Sigma}_{11}+\widetilde{\Sigma}_{12}=0$.

For $\tilde{\Sigma}_{22}$, 


$$
\begin{aligned}
& \widetilde{\Sigma}_{22}=\frac{1}{2\left(1-2 \lambda \cos \theta_{k}+\lambda^{2}\right)} \sum_{l=1}^{N-1}\left(\cos \left(l \theta_{k+j}\right)-\cos \left(l \theta_{k-j}\right)\right)-\lambda\left(\cos \left(l \theta_{k+j}-\theta_{k}\right)-\cos \left(l \theta_{k-j}-\theta_{k}\right)\right) \\
& \begin{cases}0, & k \neq j \& k+j \neq N \text { or } k=j=\frac{N}{2}, \\
-\frac{N\left(1-\lambda \cos \theta_{k}\right)}{2\left(1-2 \lambda \cos (2 \pi k / N)+\lambda^{2}\right)}, & k=j \neq \frac{N}{2}, \\
\frac{N\left(1-\lambda \cos \theta_{k}\right)}{2\left(1-2 \lambda \cos \theta_{k}+\lambda^{2}\right)}, & k+j=N \& k \neq \frac{N}{2} .\end{cases}
\end{aligned}
$$

From all of the above, it follows that

$$
\widetilde{\Sigma}_{1}+\widetilde{\Sigma}_{2}= \begin{cases}0, & k \neq j \&, k+j \neq N \text { or } k=j=\frac{N}{2}, \\ \frac{N\left(\lambda^{2}-1\right)}{2\left(1-2 \lambda \cos (2 \pi k / N)+\lambda^{2}\right)}, & k=j \neq \frac{N}{2}, \\ \frac{N\left(1-\lambda^{2}\right)}{2\left(1-2 \lambda \cos \theta_{k}+\lambda^{2}\right)}, & k+j=N \&, k \neq \frac{N}{2} .\end{cases}
$$

Therefore, we have the following.

$$
\gamma_{k, j}=E\left[\operatorname{Im}\left(\hat{x}_{k}\right) \operatorname{Im}\left(\hat{x}_{j}\right)\right]= \begin{cases}0, & k \neq j \& k+j \neq N \text { or } k=j=\frac{N}{2}, \\ \frac{\sigma^{2}}{2 N\left(1-2 \lambda \cos \theta_{k}+\lambda^{2}\right)}, & k=j \neq \frac{N}{2}, \\ -\frac{\sigma^{2}}{2 N\left(1-2 \lambda \cos \theta_{k}+\lambda^{2}\right)} & k+j=N, \& k \neq \frac{N}{2} .\end{cases}
$$

4.3. Computation of $\beta_{k, j}$. By equation (3), we have 
$E\left[\operatorname{Re}\left(\widehat{x}_{j}\right) \operatorname{Im}\left(\widehat{x}_{k}\right)\right]=-\frac{1}{N^{2}} \sum_{l, p=1}^{N-1} E\left[x_{l} x_{p}\right] \cos \left(l \theta_{j}\right) \sin \left(p \theta_{k}\right)$
Similar to the deducing process of equation (25), we obtain

$$
\begin{aligned}
& N^{2}\left(\frac{1-\lambda^{2}}{\sigma^{2}}\right) E\left[\operatorname{Re}\left(\hat{x}_{j}\right) \operatorname{Im}\left(\hat{x}_{k}\right)\right] \\
& =\sum_{l=1}^{N-1} \lambda^{l} \cos \left(l \theta_{j}\right) \sum_{p=1}^{l-1}\left(\lambda^{p}-\lambda^{-p}\right) \sin \left(s \theta_{k}\right)+\sum_{l=1}^{N-1}\left(\lambda^{l}-\lambda^{-l}\right) \cos \left(l \theta_{j}\right) \sum_{p=l}^{N-1} \lambda^{p} \sin \left(p \theta_{k}\right) \\
& =: \bar{\Sigma}_{1}+\bar{\Sigma}_{2} .
\end{aligned}
$$

Since

$$
\sum_{p=1}^{l-1}\left(\lambda^{p}-\lambda^{-p}\right) \sin \left(p \theta_{k}\right)=\bar{\mu}_{l}^{1}+\bar{\mu}_{l}^{2}
$$

$$
\begin{aligned}
& \bar{\mu}_{l}^{1}=-\lambda^{l} \frac{\sin \left(l \theta_{k}\right)-\lambda \sin (l-1) \theta_{k}}{1-2 \lambda \cos \theta_{k}+\lambda^{2}}, \\
& \bar{\mu}_{l}^{2}=\lambda^{-l+2} \frac{\sin \left(l \theta_{k}\right)-\lambda^{-1} \sin (l-1) \theta_{k}}{1-2 \lambda \cos \theta_{k}+\lambda^{2}},
\end{aligned}
$$

where

we have

$$
\begin{aligned}
\bar{\Sigma}_{1}= & -\frac{1}{1-2 \lambda \cos \theta_{k}+\lambda^{2}} \sum_{l=1}^{N-1} \lambda^{2 l} \cos \left(l \theta_{j}\right)\left(\sin \left(l \theta_{k}\right)-\lambda \sin (l-1) \theta_{k}\right) \\
& +\frac{\lambda^{2}}{1-2 \lambda \cos \theta_{k}+\lambda^{2}} \sum_{l=1}^{N-1} \cos \left(l \theta_{j}\right)\left(\sin \left(l \theta_{k}\right)-\lambda^{-1} \sin (l-1) \theta_{k}\right)=: \bar{\Sigma}_{11}+\bar{\Sigma}_{12} .
\end{aligned}
$$

Furthermore,

$$
\bar{\Sigma}_{12}=\frac{\lambda^{2}}{2\left(1-2 \lambda \cos \theta_{k}+\lambda^{2}\right)} \sum_{l=1}^{N-1}\left(\sin \left(l \theta_{k+j}\right)+\sin \left(l \theta_{k-j}\right)-\lambda^{-1}\left(\sin \left(l \theta_{k+j}-\theta_{k}\right)+\sin \left(l \theta_{k-j}-\theta_{k}\right)\right)\right) .
$$

Noticing that $\sum_{l=1}^{N-1} \sin \left(l \theta_{k+j}\right)=\sum_{l=1}^{N-1} \sin \left(l \theta_{k-j}\right)=0$, we obtain

$$
\bar{\Sigma}_{12}=-\frac{\lambda}{2\left(1-2 \lambda \cos \theta_{k}+\lambda^{2}\right)} \sum_{l=1}^{N-1}\left(\sin \left(l \theta_{k+j}-\theta_{k}\right)+\sin \left(l \theta_{k-j}-\theta_{k}\right)\right)
$$

For $\bar{\Sigma}_{2}$, we obtain 


$$
\begin{aligned}
\bar{\Sigma}_{2}= & \frac{1}{1-2 \lambda \cos \theta_{k}+\lambda^{2}} \sum_{l=1}^{N-1} \lambda^{2 l} \cos \left(l \theta_{j}\right)\left(\sin \left(l \theta_{k}\right)-\lambda \sin (l-1) \theta_{k}\right) \\
& -\frac{1}{1-2 \lambda \cos \theta_{k}+\lambda^{2}} \sum_{l=1}^{N-1} \cos \left(l \theta_{j}\right)\left(\sin \left(l \theta_{k}\right)-\lambda \sin (l-1) \theta_{k}\right)=: \bar{\Sigma}_{21}+\bar{\Sigma}_{22},
\end{aligned}
$$

where

$$
\begin{gathered}
\bar{\Sigma}_{22}=-\frac{1}{2\left(1-2 \lambda \cos \theta_{k}+\lambda^{2}\right)} \sum_{l=1}^{N-1}\left(\sin \left(l \theta_{k+j}\right)+\sin \left(l \theta_{k-j}\right)-\lambda\left(\sin \left(l \theta_{k+j}-\theta_{k}\right)+\sin \left(l \theta_{k-j}-\theta_{k}\right)\right)\right) \\
=\frac{\lambda}{2\left(1-2 \lambda \cos \theta_{k}+\lambda^{2}\right)} \sum_{l=1}^{N-1}\left(\sin \left(l \theta_{k+j}-\theta_{k}\right)+\sin \left(l \theta_{k-j}-\theta_{k}\right)\right) . \\
\beta_{k, j}=E\left[\operatorname{Re}\left(\hat{x}_{k}\right) \operatorname{Im}\left(\hat{x}_{j}\right)\right]=0, \quad k, j=0, \ldots, N-1 .
\end{gathered}
$$

Since

$$
\begin{aligned}
& \bar{\Sigma}_{11}+\bar{\Sigma}_{21}=0, \\
& \bar{\Sigma}_{12}+\bar{\Sigma}_{22}=0,
\end{aligned}
$$

we have the following.

4.4. Proof of Theorem 1. Without loss of generalization, we assume that $N$ is an odd number. By equation (4), we obtain

Lemma 3. When $\lambda^{N} \approx 0$, the series $E\left[\left\{\operatorname{Re}\left(\widehat{x}_{k}\right), \operatorname{Im}\right.\right.$ $\left.\left.\left(\widehat{x}_{j}\right)\right\}\right]_{k, j=0, \ldots, N-1}$ satisfy

$$
\begin{aligned}
\operatorname{Re}\left(W_{n}(s)\right) & =\left(\sum_{k=0}^{[N / 2]}+\sum_{k=[N / 2]+1}^{N-1}\right) r_{k}\left(\operatorname{Re}\left(\widehat{x}_{k}\right) \cos \eta_{n, k}-\operatorname{Im}\left(\widehat{x}_{k}\right) \sin \eta_{n, k}\right) \\
& =I_{1}+I_{2},
\end{aligned}
$$

where

$$
\begin{aligned}
\eta_{n, k} & =\varphi_{k}+n \theta_{k}, \\
\varphi_{k} & =\operatorname{Arg}\left(\overline{\widehat{\psi}}\left(s \omega_{k}\right)\right), \\
r_{k} & =\left|\widehat{\psi}\left(s \omega_{k}\right)\right| .
\end{aligned}
$$

$$
\begin{aligned}
\operatorname{Re}\left(\widehat{x}_{N-k}\right) & =\operatorname{Re}\left(\widehat{x}_{k}\right), \\
\operatorname{Im}\left(\widehat{x}_{N-k}\right) & =-\operatorname{Im}\left(\widehat{x}_{k}\right) .
\end{aligned}
$$

Since $\hat{x}_{k}$ is real, we have $\overline{\hat{x}}_{k}=\hat{x}_{N-k}$. This implies that

Since $\psi$ is real-valued, $\widehat{\psi}(\xi)=\overline{\widehat{\psi}}(-\xi)$; by $\omega_{k}=\left(\theta_{k} / \delta t\right)$, $k \leq(N / 2)$, and $\omega_{k}=-\left(\theta_{N-k} / \delta t\right), k>(N / 2)$, we obtain

$$
\widehat{\psi}\left(s \omega_{N-k}\right)=\widehat{\psi}\left(-s \omega_{k}\right)=\bar{\psi}\left(s \omega_{k}\right) .
$$

Therefore, by equation (77),

$$
\begin{aligned}
r_{N-k} & =\left|\widehat{\psi}\left(s \omega_{N-k}\right)\right|=\left|\widehat{\psi}\left(s \omega_{k}\right)\right|=r_{k}, \\
\varphi_{N-k} & =\operatorname{Arg}\left(\overline{\widehat{\psi}}\left(s \omega_{N-k}\right)\right)=\operatorname{Arg}\left(\overline{\widehat{\psi}}\left(s \omega_{k}\right)\right)=-\operatorname{Arg}\left(\widehat{\psi}\left(s \omega_{k}\right)\right)=-\varphi_{k} .
\end{aligned}
$$

Hence, $\quad \eta_{n, N-k}=\varphi_{N-k}+n \theta_{N-k}=-\varphi_{k}-n \theta_{k}=-\eta_{n, k}$.

From this, $\widehat{\psi}(0)=0$, and equation (76), it follows that 


$$
\begin{aligned}
I_{2} & =\sum_{k=[N / 2]+1}^{N-1} r_{k}\left(\operatorname{Re}\left(\widehat{x}_{k}\right) \cos \eta_{n, k}-\operatorname{Im}\left(\widehat{x}_{k}\right) \sin \eta_{n, k}\right) \\
& =\sum_{k=1}^{[N / 2]} r_{N-k}\left(\operatorname{Re}\left(\widehat{x}_{N-k}\right) \cos \eta_{n, N-k}-\operatorname{Im}\left(\widehat{x}_{N-k}\right) \sin \eta_{n, N-k}\right) \\
& =I_{1}, \\
\left(W_{n}(s)\right)^{2} & =4 \sum_{k, j=0}^{[N / 2]} r_{k} r_{j}\left(\operatorname{Re}\left(\widehat{x}_{k}\right) \cos \eta_{n, k}-\operatorname{Im}\left(\widehat{x}_{k}\right) \sin \eta_{n, k}\right)\left(\operatorname{Re}\left(\widehat{x}_{j}\right) \cos \eta_{n, j}-\operatorname{Im}\left(\hat{x}_{j}\right) \sin \eta_{n, j}\right) .
\end{aligned}
$$

By Lemmas 1-3, we obtain

$$
\begin{aligned}
E\left[\left(W_{n}(s)\right)^{2}\right] & =4 \sum_{k=0}^{[N / 2]} r_{k}^{2}\left(\left(E\left[\operatorname{Re}\left(\widehat{x}_{k}\right)\right]\right)^{2} \cos ^{2} \eta_{n, k}+\left(E\left[\operatorname{Im}\left(\widehat{x}_{k}\right)\right]\right)^{2} \sin ^{2} \eta_{n, k}\right) \\
& =4 \sum_{k=0}^{[N / 2]} r_{k}^{2}\left(\operatorname{Re}\left(\widehat{x}_{k}\right)\right)^{2}\left(\cos ^{2} \eta_{n, k}+\sin ^{2} \eta_{n, k}\right) \\
& =4 \sum_{k=0}^{[N / 2]} r_{k}^{2}\left(\operatorname{Re}\left(\widehat{x}_{k}\right)\right)^{2} \\
& =\frac{2 \sigma^{2}}{N} \sum_{k=0}^{[N / 2]} \frac{\left|\widehat{\psi}\left(s \omega_{k}\right)\right|^{2}}{1-2 \lambda \cos \theta_{k}+\lambda^{2}} .
\end{aligned}
$$

Since $W_{n}(s)=\operatorname{Re}\left(W_{n}(s)\right)$ is a Gaussian random variable with mean 0 , we obtain Theorem 1 .

4.5. Proof of Theorems 2 and 3. Without loss of generalization, we assume that $N$ is an old number. By equation (4), for $n=0, \ldots, N-1$, we have

$$
W_{n}(s)=\sum_{k=0}^{[N / 2]} \widehat{x}_{k} \overline{\widehat{\psi}}\left(s \omega_{k}\right) e^{i n \theta_{k}}+\sum_{k=[N / 2]+1}^{N-1} \widehat{x}_{k} \overline{\widehat{\psi}}\left(s \omega_{k}\right) e^{i n \theta_{k}}
$$

Since $\psi$ is an analytic wavelet, $\widehat{\psi}(\omega)=0, \omega<0$. Notice that $\omega_{k}<0, k>(N / 2)$. The second term on the right-hand side of equation (83) is vanishing. Therefore,

$$
E\left[\left(\operatorname{Re}\left(W_{n}(s)\right)\right)^{2}\right]=\sum_{k, j=0}^{[N / 2]} r_{k} r_{j}\left(E\left[\operatorname{Re}\left(\widehat{x}_{k}\right) \operatorname{Re}\left(\widehat{x}_{j}\right)\right] \cos \eta_{n, k} \cos \eta_{n, j}+E\left[\operatorname{Im}\left(\widehat{x}_{k}\right) \operatorname{Im}\left(\widehat{x}_{j}\right)\right] \sin \eta_{n, k} \sin \eta_{n, j}\right)
$$

By Lemmas 1 and 2, $E\left[\left(\operatorname{Re}\left(\hat{x}_{k}\right)\right)^{2}\right]=E\left[\left(\operatorname{Im}\left(\hat{x}_{k}\right)\right)^{2}\right]$, $0 \leq k \leq[N / 2]$, and so 


$$
\begin{aligned}
& E\left[\left(\operatorname{Re}\left(W_{n}(s)\right)\right)^{2}\right] \\
& =\sum_{k=0}^{[N / 2]} r_{k}^{2}\left(E\left[\left(\operatorname{Re}\left(\widehat{x}_{k}\right)\right)^{2}\right] \cos ^{2} \eta_{n, k}+E\left[\left(\operatorname{Im}\left(\widehat{x}_{k}\right)\right)^{2}\right] \sin ^{2} \eta_{n, k}\right) \\
& =\sum_{k=0}^{[N / 2]} r_{k}^{2} E\left[\left(\operatorname{Re}\left(\hat{x}_{k}\right)\right)^{2}\right]\left(\cos ^{2} \eta_{n, k}+\sin ^{2} \eta_{n, k}\right)=\sum_{k=0}^{[N / 2]} r_{k}^{2} E\left[\left(\operatorname{Re}\left(\widehat{x}_{k}\right)\right)^{2}\right] .
\end{aligned}
$$

Similarly, we have

By Lemma 3,

$E\left[\left(\operatorname{Im}\left(W_{n}(s)\right)\right)^{2}\right]=\sum_{k, j=0}^{[N / 2]} r_{k} r_{j} E\left[\operatorname{Im}\left(\widehat{x}_{k} e^{i \eta_{n, k}}\right) \operatorname{Im}\left(\hat{x}_{j} e^{i \eta_{n, j}}\right)\right]$.

$$
E\left[\operatorname{Im}\left(\widehat{x}_{k} e^{i \eta_{n, k}}\right) \operatorname{Im}\left(\hat{x}_{j} e^{i \eta_{n, j}}\right)\right]=E\left[\operatorname{Re}\left(\widehat{x}_{k}\right) \operatorname{Re}\left(\widehat{x}_{j}\right)\right] \sin \eta_{n, k} \sin \eta_{n, j}+E\left[\operatorname{Im}\left(\widehat{x}_{k}\right) \operatorname{Im}\left(\hat{x}_{j}\right)\right] \cos \eta_{n, k} \cos \eta_{n, j} .
$$

This implies that

$$
E\left[\left(\operatorname{Im}\left(W_{n}(s)\right)\right)^{2}\right]=\sum_{k=0}^{N / 2-1} r_{k}^{2}\left(E\left[\left(\operatorname{Re}\left(\widehat{x}_{k}\right)\right)^{2}\right] \sin ^{2} \eta_{n, k}+E\left[\left(\operatorname{Im}\left(\widehat{x}_{k}\right)\right)^{2}\right] \cos ^{2} \eta_{n, k}\right)=\sum_{k=0}^{(N / 2)-1} r_{k}^{2} E\left[\left(\operatorname{Re}\left(\widehat{x}_{k}\right)\right)^{2}\right] .
$$

Therefore, we have

$$
\operatorname{Var}\left(\operatorname{Re}\left(W_{n}(s)\right)\right)=\operatorname{Var}\left(\operatorname{Im}\left(W_{n}(s)\right)\right)=\sum_{k=0}^{[N / 2]} r_{k}^{2} E\left[\left(\operatorname{Re}\left(\widehat{x}_{k}\right)\right)^{2}\right]=\frac{\sigma^{2}}{2 N\left(1-\lambda^{2}\right)} \sum_{k=0}^{[N / 2]} P_{k}(\lambda)\left|\widehat{\psi}\left(s \omega_{k}\right)\right|^{2}
$$

Now, we prove that $\operatorname{Re}\left(W_{n}(s)\right)$ and $\operatorname{Im}\left(W_{n}(s)\right)$ are independent.

By Lemmas 1-3,

$$
\begin{aligned}
& E\left[\operatorname{Re}\left(W_{n}(s)\right) \operatorname{Im}\left(W_{n}(s)\right)\right] \\
& =\sum_{k, j=0}^{[N / 2]} r_{k} r_{j}\left(E\left[\operatorname{Re}\left(\widehat{x}_{k}\right) \operatorname{Re}\left(\widehat{x}_{j}\right)\right] \cos \eta_{n, k} \sin \eta_{n, j}-E\left[\operatorname{Im}\left(\widehat{x}_{k}\right) \operatorname{Im}\left(\widehat{x}_{j}\right)\right] \sin \eta_{n, k} \cos \eta_{n, j}\right) \\
& =\frac{1}{2} \sum_{k=0}^{[N / 2]} r_{k}^{2}\left(E\left[\left(\operatorname{Re}\left(\widehat{x}_{k}\right)\right)^{2}\right] \sin \left(2 \eta_{n, k}\right)-E\left[\left(\operatorname{Im}\left(\widehat{x}_{k}\right)\right)^{2}\right] \sin \left(2 \eta_{n, k}\right)\right)=0 .
\end{aligned}
$$

Since both $\operatorname{Re}\left(W_{n}(s)\right)$ and $\operatorname{Im}\left(W_{n}(s)\right)$ are Gaussian random variables with mean 0 and

$$
\operatorname{Var}\left(\operatorname{Re}\left(W_{n}(s)\right)\right)=\operatorname{Var}\left(\operatorname{Im}\left(W_{n}(s)\right)\right)=: \sigma^{2}(s),
$$
where

$$
\sigma^{2}(s)=\frac{\sigma^{2}}{2 N\left(1-\lambda^{2}\right)}\left(\sum_{k=0}^{[N / 2]} P_{k}(\lambda)\left|\widehat{\psi}\left(s \omega_{k}\right)\right|^{2}\right),
$$

we obtain Theorem 2.

Consider the phase $\Phi=\operatorname{Arg}\left(W_{n}(s)\right)$. Notice that the p.d.f.s of $\operatorname{Re}\left(W_{n}(s)\right)$ and $\operatorname{Im}\left(W_{n}(s)\right)$ are, respectively, 


$$
\begin{aligned}
& p_{R}(x)=\frac{1}{\sqrt{2 \pi} \sigma(s)} e^{-\left(x^{2} / 2 \sigma^{2}(s)\right)}, \\
& p_{I}(x)=\frac{1}{\sqrt{2 \pi} \sigma(s)} e^{-\left(y^{2} / 2 \sigma^{2}(s)\right)},
\end{aligned}
$$

and $\operatorname{Re}\left(W_{n}(s)\right)$ and $\operatorname{Im}\left(W_{n}(s)\right)$ are independent, the joint p.d.f. of $\operatorname{Re}\left(W_{n}(s)\right)$ and $\operatorname{Im}\left(W_{n}(s)\right)$ is

$$
p(x, y)=\frac{1}{2 \pi \sigma^{2}(s)} e^{-\left(\left(x^{2}+y^{2}\right) / 2 \sigma^{2}(s)\right)} .
$$

The probability

$$
P\left(\beta_{1}<\Phi<\beta_{2}\right)=\frac{1}{2 \pi \sigma^{2}(s)} \iint_{\Omega} e^{-\left(\left(x^{2}+y^{2}\right) / 2 \sigma^{2}(s)\right)} \mathrm{d} x \mathrm{~d} y,
$$

where the domain $\Omega:=\left\{0<r<\infty, 0 \leq \beta_{1}<\varphi<\beta_{2}<2 \pi\right\}$. Hence,

$$
P\left(\beta_{1}<\Phi<\beta_{2}\right)=\frac{1}{2 \pi \sigma^{2}(s)} \int_{\beta_{1}}^{\beta_{2}} \mathrm{~d} \varphi \int_{0}^{\infty} r e^{-\left(r^{2} / 2 \sigma^{2}(s)\right)} \mathrm{d} r=\frac{1}{2 \pi}\left(\beta_{2}-\beta_{1}\right),
$$

i.e., Theorem 3 holds.

\section{Climate Diagnosis}

Climatic background noises are often modeled as red noise [4-9]. In order to extract instinct features of climatic time series, we can use Theorems 1 and 2 to compare the wavelet power spectrum of climatic time series with that of red noise. If the values of the wavelet power spectrum of climate time series at some regions are all outside the $95 \%$ confidence interval for the distribution for the wavelet power spectrum of the red noise, then the wavelet power spectrum at this region contain instinct features of climatic time series. Moreover, we can use Theorem 3 to test the synchronization between the two climatic time series. If the difference of the wavelet phase of the two climatic time series does not change in some significant regions of the wavelet power spectrum, noticing that Theorem 3 indicates that this cannot be caused by climatic background noise effects, these two climatic time series demonstrate synchronization.

\section{Data Availability}

No data were used to support this research.

\section{Conflicts of Interest}

The author declares no conflicts of interest.

\section{Acknowledgments}

This research was partially supported by the European Commissions Horizon 2020 Framework Program under No. 861584 and Taishan Distinguished Professor Fund.

\section{References}

[1] R. N. McDonough and A. D. Whalen, Detection of Signals in Noise, Academic Press, Cambridge, MA, USA, 1995.

[2] T. Li, Time Series with Mixed Spectra, Chapman and Hall/ CRC, Boca Raton, FL, USA, 2019.

[3] L. Abualigah and A. Diabat, "Advances in sine cosine algorithm: a comprehensive survey," Artificial Intelligence Review, vol. 54, no. 4, pp. 2567-2608, 2021.

[4] Z. Zhang, Multivariate Time Series Analysis in Climate and Environmental Research, Springer, Berlin, Germany, 2018.

[5] Z. Zhang, Big Data Mining for Climate Change, Elsevier, Amsterdam, Netherlands, 2020.

[6] M. E. Mann and J. M. Lees, "Robust estimation of background noise and signal detection in climatic time series," Climatic Change, vol. 33, no. 3, pp. 409-445, 1996.

[7] M. R. Allen and L. A. Smith, "Investigating the origins and significance of low-frequency modes of climate variability," Geophysical Research Letters, vol. 21, no. 10, pp. 883-886, 1994.

[8] J. E. Kutzbach and R. A. Bryson, "Variance spectrum of holocene climatic fluctuations in the north atlantic sector," Journal of the Atmospheric Sciences, vol. 31, no. 8, pp. 19581963, 1974.

[9] D. L. Gilman, F. J. Fuglister, and J. M. Mitchell, "On the power spectrum of "red noise"," Journal of the Atmospheric Sciences, vol. 20, no. 2, pp. 182-184, 1963.

[10] L. Abualigah and M. Alkhrabsheh, "Amended hybrid multiverse optimizer with genetic algorithm for solving task scheduling problem in cloud computing," The Journal of Supercomputing, 2021.

[11] L. Abualigaha, A. Diabat, S. Mirjalili, M. A. Elaziz, and A. H. Gandomi, "The arithmetic optimization algorithm," Computer Methods in Applied Mechanics and Engineering, vol. 376, Article ID 113609, 2021.

[12] L. Abualigah, D. Yousri, M. A. Elaziz, A. A. Ewees, M. A. A. Al-qaness, and A. H. Gandomi, "Aquila optimizer: a novel meta-heuristic optimization algorithm," Computers \& Industrial Engineering, vol. 157, Article ID 107250, 2021.

[13] L. Abualigah, A. Diabat, and M. A. Elaziz, "Intelligent workflow scheduling for big data applications in IoT cloud computing environments," Cluster Computing, 2021.

[14] C. K. Chui, An Introduction to Wavelet, Academic Press, Cambridge, MA, USA, 1992.

[15] C. Torrence and G. P. Compo, "A practical guide to wavelet analysis," Bulletin of the American Meteorological Society, vol. 79, no. 1, pp. 61-78, 1998.

[16] M. Jansen, Noise Reduction by Wavelet Thresholding, Vol. 161, Springer-Verlag, New York, NY, USA, 1 edition, 2001.

[17] D. L. Donoho, "De-noising by soft thresholding," IEEE Transactions on Information Theory, vol. 41, no. 3, Article ID 613C627, 1995.

[18] G. P. Nason and D. Savchev, "White noise testing using wavelets," Stat, vol. 3, no. 1, pp. 351-362, 2014.

[19] G. Ferrante and D. Persano Adorno, "A wavelet analysis of $1 / \mathrm{f}$ and white noise in microwave transistors," Microelectronics Reliability, vol. 41, no. 1, pp. 99-104, 2001.

[20] Z. Zhang and P. Jorgensen, "Modulated haar wavelet analysis of climatic background noise," Acta Applicandae Mathematica, vol. 140, pp. 71-93, 2015. 